\title{
A Study on Optimizing the Riveting of Steel Balls for Hand Tools
}

\author{
Ying-Hsiang Lin ${ }^{1, a}$, Shang-Pin $\mathrm{Li}^{1, \mathrm{~b}}$, Sung-Chin Huang ${ }^{1, \mathrm{c}}$, \\ Chien-Yu Lu ${ }^{1, d}$ and Chih-Cheng Huang ${ }^{2, e,{ }^{*}}$ \\ 1 Department of Industrial Education and Technology, National Changhua University of Education, \\ Changhua, Taiwan, R.O.C \\ ${ }^{2}$ Department of Leisure \& Recreation, National Formosa University, Yunlin, Taiwan, R.O.C \\ as3150316@gmail.com, bspli@cyut.edu.tw, c jilihondao@gmail.com, d33150316@yahoo.com.tw, \\ ecchuang@nfu.edu.tw \\ * The Corresponding author
}

Keywords: Hand tool steel ball riveting; Tightness; Taguchi's Quality Engineering

\begin{abstract}
Steel ball riveting mainly functions to attach sockets for hand tools. The connectivity between sockets and accessories is directly linked to how tight the steel balls are riveted: if too tight, then it will be difficult to change the accessories or they will even jam; otherwise, they will loosen themselves off. Improving the quality of steel balls lies in controlling related parameters in the process. The paper proposes a model to optimize the riveting of steel balls based on better refining a hand tool manufacturer's riveting process. The study probes the optimized combination of parameters for riveting on the experimental level by combining the design methods of TRIZ and Taguchi's Quality Engineering. By increasing Cpk from 0.45 to 1.46, the results reveal that the process capability index registers a dramatic rise to the required precision, thus improving the quality of steel ball riveting and the stability of its process capability.
\end{abstract}

\section{Introduction}

The hand tools industry produces products with various applications in various categories, but mostly involves equipment maintenance as the main application of Taiwan's hand tool industry. The DIY market, as well as agriculture, forestry, and horticulture, have witnessed numerous applications. While the classification of hand tool products varies from manufacturer to manufacturer, it is mainly divided into two categories of non-powered hand tools and powered hand tools, as based on different working environments, characteristics, industries, modes of operation, and use. The wrench and socket occupy a very important position in the output of hand tool exports and in today's industry. In addition, the quality of hand tools will affect the downstream workstations, as well as the aesthetic value of the product's appearance. This study probes into the quality of steel ball riveting in many industries, improves the settings of control parameters, and further enhances the yield of steel ball riveting size and tightness.

The Theory of Innovative (Inventive) Problem Solving is referred to as TRIZ [1]. Many current studies have explored TRIZ [2] [3]. The Taguchi Method, which can be applied to a wide range of experimental trend analysis with a small number of experimental data, can effectively improve manufacturing processes and process quality. There are many scholars devoted to the study of Taguchi research areas [4] [5] [6]. This study aims to improve analysis, and proposes suggestions according to the study of process capability combining the optimized parameters of steel ball riveting process quality for hand tool manufacturers and Taguchi Quality Engineering, in order to reduce the degree of process variation, improve the stability of product quality, and improve process capability and stability. Finally, the data will be provided to manufacturers of hand tools, and beyond, as the basis of stable production. The aims of this study are as follows:

1. This study explores the main factors that affect the stability of hand tool steel ball riveting size during the manufacturing process. 
2. This study explores the impact of the process capability of hand tool steel ball riveting size, as well as the optimization of process parameters, as the basis for controlling and improving the size of hand tool steel ball riveting.

\section{Literature References}

Hand Tool Steel Ball Riveting. Hand tool steel ball riveting (as illustrated in Fig. 1) mainly enables users to conveniently and quickly change the required specifications and size when using hand tools, which correspond to the screw, nut, socket, or screwdriver of other work ends in maintenance or operating environments, in order to carry out repairs or adjustments. The height of the steel ball must meet the requirements of the customer, and then, meet the requirements of weight. Only hand tools produced according to the standard can simultaneously meet the required functions and quality stability, as required by customers.

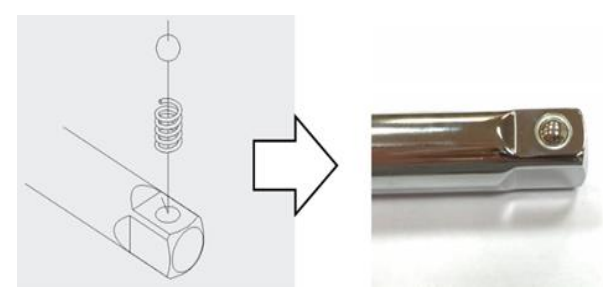

Figure 1. Hand tool steel ball riveting

TRIZ. The Theory of Innovative (Inventive) Problem Solving is referred to as TRIZ [1]. The problem-solving tools of TRIZ include 39 engineering parameters, 76 standard solutions, 40 invention principles, Algorithm for Inventive Problem Solution (ARIZ), substance-field analysis, evolutionary trends, and scientific effects.

Taguchi's Quality Engineering. The Taguchi Method originated in 1950 from the advocacy of Taguchi. It is an experimental plan conducive to improving product quality, and generally applies more multi-factor orthogonal experimental designs. The biggest advantage of this method is that it can conduct extensive experimental trend analysis with only a small amount of experimental data to effectively improve manufacturing processes and process quality. The principles, experimental processes, and restrictions are described, as follows [7] [8]:

Loss Function. According to Taguchi, quality loss is measured by the characteristics of demand and expected performance. The loss function relates to the deviation of a product's quality characteristics from the target value: the loss is zero when the quality characteristics meet the target value; loss emerges if the characteristics deviate from the target value. The function of the Taguchi Method propels manufacturers to continue to pursue quality improvements by filling the gap between the quality characteristic and the target value.

Quality Characteristic. The key to the first step in the Taguchi Method analysis process is to determine the objective function and its characteristics. The greatest feature is the conversion ability of the objective function into the $\mathrm{S} / \mathrm{N}$ ratio calculation method for subsequent analysis steps.

Definition of the control factor and the level table. Before the analysis of the Taguchi Method, the level of each control factor must be set in the objective function.

Selection of orthogonal array. An orthogonal array is applied to experimental data analysis to reduce the number of analyses, while ensuring accuracy.

Analysis of Variance. It mainly assesses the relative importance of each control factor and its experimental error, in which the Percent Contribution aims to understand the ratio of individual difference or error. 


\section{Case Study}

Define. In hand tools, a socket in need of steel ball riveting often encounters instability in quality or improper tightness (overly loose or tight) due to the varying heights of the steel balls. In a severe case, steel balls may loosen or become jammed. Only after meeting the customer's requirements, and improving the satisfaction and stability of the product's size, can the reject rate be reduced and the expected effects be achieved.

Measure. Yield analysis uses the $\mathrm{C}_{\mathrm{pk}}$ value of Process Capability Indices to present actual capability. Process Capability Indices assume that the processes of known quality factors are controlled by the quality capability presented in the normal state, which correspond to the assessment of three main materials: specifications of characteristics, the central position of process characteristics, and the degree of consistency, in order to describe the deviation from the process center (process accuracy) and process consistency (process precision). The general process capability is based on $\mathrm{C}_{\mathrm{pk}}$ as the evaluation index, where $\mathrm{C}_{\mathrm{pk}} \geqq 1.33$ denotes qualified, $1 \leqq \mathrm{C}_{\mathrm{pk}}<1.33$ denotes warning, and $\mathrm{C}_{\mathrm{pk}}$ $<1$ denotes unqualified [9].

The experimented manufacturer randomly selects 100 pieces of products in production in order to measure the height of steel ball riveting, as illustrated in Fig. 2.

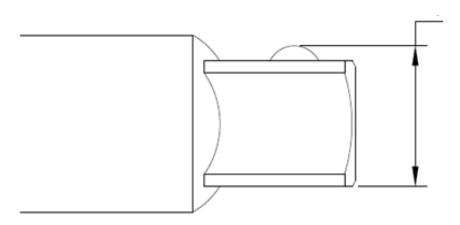

Figure 2 Height of steel ball riveting

PCI is calculated as $\mathrm{C}_{\mathrm{pk}}=0.45<1.33$ by Minitab software, indicating that the process is unstable and the process capability is insufficient. As a result, there is still room for improvement and progress in the overall process (as illustrated in Fig. 3).

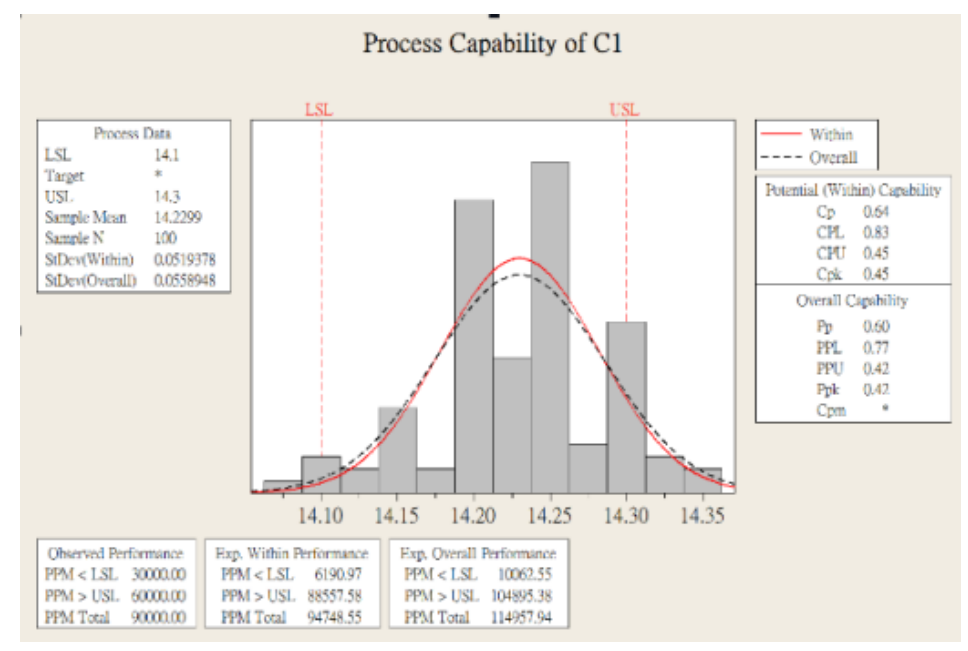

Figure 3. Current PCI Analyze

Analyze. The technical contradiction in this study conducting analysis of steel ball riveting size, as based on the current situation and TRIZ, lies in: parameter 13 "object stability" and parameter 4 "fixed length". Principles 37 and 39 are ignored in this study; while principle 35 "parameter change" of the invention is:

Change physical state; Change concentration or density; Change the degree of elasticity; Change the temperature; Change pressure and Change length and volume. 
Selection of the Experimental Factor and Level. After filtering factors, the control factor and level factor are 3, as obtained using an L9 orthogonal table (see Table 1).

Table 1 Control factor and level

\begin{tabular}{|c|c|c|c|c|}
\hline & Factor & Level 1 & Level 2 & Level 3 \\
\hline A & drilling size & $\varnothing 6.4$ & $\varnothing 6.5$ & $\varnothing 6.6$ \\
\hline B & hydraulic pressure & $46 \mathrm{~kg} / \mathrm{cm} 2$ & $49 \mathrm{~kg} / \mathrm{cm} 2$ & $52 \mathrm{~kg} / \mathrm{cm} 2$ \\
\hline C & punch size & $8 \mathrm{~mm}$ & $8.5 \mathrm{~mm}$ & $9 \mathrm{~mm}$ \\
\hline
\end{tabular}

\section{Improve.}

Determination of the Experimental Method and Factor Configuration. Taguchi argued that the objective function should be defined based on the quality that requires improvement, the factors and levels affecting the objective function should be identified, and an orthogonal table should be used to determine the factor configuration and number of experiments in order to obtain the same information as obtained from the full-factor experiment, while reducing the number. Considering cost-effectiveness, this study adopts the Taguchi Method to determine the best combination of management levels. It is judged that the interaction between the experimental factors is so small that the effect can be regarded as part of the experimental error.

The experiment uses the 3 factors and 3 levels, thus, the L9 orthogonal array is chosen regardless of the interaction.

Analysis of experimental data. The experimental response and $\mathrm{S} / \mathrm{N}$ ratio are calculated by Minitab software after results are obtained from the experiment with the experimental factors and level planning of the Taguchi experimental design.

Table 2 Experimental response of orthogonal arrays and $\mathrm{S} / \mathrm{N}$ ratio

\begin{tabular}{|c|c|c|c|c|c|c|c|c|}
\hline data & A & B & C & 1 & 2 & 3 & 4 & SNRA \\
\hline 1 & 1 & 1 & 1 & 14.28 & 14.24 & 14.17 & 14.28 & 48.7715 \\
\hline 2 & 1 & 2 & 2 & 14.27 & 14.29 & 14.19 & 14.2 & 49.1038 \\
\hline 3 & 1 & 3 & 3 & 14.32 & 14.34 & 14.36 & 14.31 & 56.2097 \\
\hline 4 & 2 & 1 & 2 & 14.24 & 14.11 & 14.26 & 14.24 & 46.2789 \\
\hline 5 & 2 & 2 & 3 & 14.36 & 14.02 & 14.28 & 14.38 & 38.6943 \\
\hline 6 & 2 & 3 & 1 & 14.44 & 14.18 & 14.29 & 14.25 & 42.2848 \\
\hline 7 & 3 & 1 & 3 & 14.21 & 14.08 & 14.15 & 14.3 & 43.6414 \\
\hline 8 & 3 & 2 & 1 & 14.24 & 14.07 & 14.31 & 14.18 & 42.9034 \\
\hline 9 & 3 & 3 & 2 & 14.32 & 14.2 & 14.25 & 14.06 & 42.2255 \\
\hline
\end{tabular}

The larger the $\mathrm{S} / \mathrm{N}$ ratio, the better the combination of parameters, as a larger $\mathrm{S} / \mathrm{N}$ ratio indicates better quality (smaller loss). Products manufactured within this parameter level have the smallest variation (as shown in Fig. 4 ).

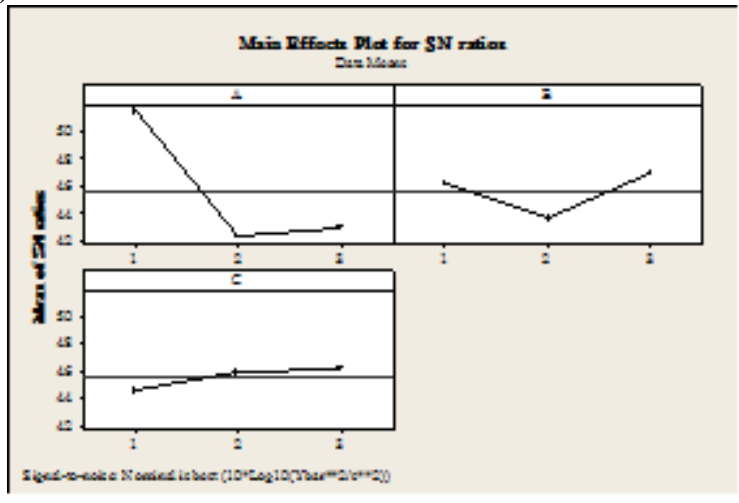

Figure 4. S/N ratio effect 
The best combination of parameters is A1-B3-C3, as indicated in the $\mathrm{S} / \mathrm{N}$ ratio. A: working drilling size $\varnothing 6.4 \mathrm{~mm}$, B: hydraulic pressure $52 \mathrm{~kg} / \mathrm{cm}$, punch size $9 \mathrm{~mm}$ (As shown in Table 3 ).

Table 3 Average $\mathrm{S} / \mathrm{N}$ ratio of each factor level

\begin{tabular}{|c|c|c|c|}
\hline Level & A & B & C \\
\hline 1 & 51.36 & 46.23 & 44.65 \\
\hline 2 & 42.42 & 43.57 & 45.87 \\
\hline 3 & 42.92 & 46.91 & 46.18 \\
\hline Effect & 8.94 & 3.34 & 1.53 \\
\hline
\end{tabular}

There are 80 responses obtained from verification, which are calculated for PCI with a Cpk of 1.46, larger than the original 0.45 , and better than the qualification standard $\mathrm{Cpk}=1.33$, which indicates that the A1-B3-C3 combination in this study can indeed improve the process capability of hand tool steel ball riveting.

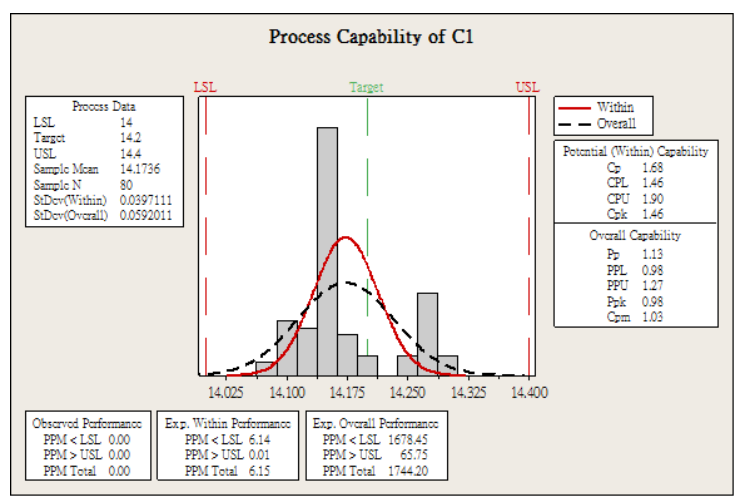

Figure 5. Process capability of results

\section{Conclusions}

This study uses TRIZ and the Taguchi Method to determine the best parameters for the riveting and processing of hand tool steel balls, and drastically improves the Process Capability Index ( Cpk) from 0.45 to 1.46 , showing that this combination can effectively improve hand tool steel ball riveting.

The key point of the study is to first conduct analysis with TRIZ, and then, the Taguchi Method, in order to identify the feasible optimum parameters, followed by experiments to ensure feasibility. After verification, the optimum combination of parameters can effectively improve the efficiency of the process, and provide reference for domestic hand tool steel ball riveting and factories.

\section{References}

[1] M.H. Sung, Systematic innovation: An introduction to TRIZ, Tingmao Publish Co., 2009.

[2] Lin, Y.H., Li, S.P., Lu, C.Y., Chung, R.G., Chen, D.F., Research into the Improvement on Hexagonal Socket by Applying the System Innovation Theory Advances in Engineering Research, Vols. 52 (2016), p. 55-57.

[3] Chang, C. P., Lin, Y.H. and Lu, C. Y., Applying TRIZ Systematic Innovation Method to Improve Urinals, Advances in Engineering Research, Vols. 22 (2015), p.793-795.

[4] Lee, R.S., Bakthavatsalam, R., Marsh, C.L. and Kuhr, C.S., Ureteral Complications in Renal Transplantation: A Comparison of the Lich-Gregoir Versus the Taguchi Technique, Transplantation Proceedings, Volume: 39, Issue: 5 (2007), p.1461-1464.

[5] Hong, C. W., Using the Taguchi method for effective market segmentation, Expert Systems With Applications, Volume 39, Issue 5 (2012), p. 5451-5459.

[6] Chang, C. P., Lin, Y.H. and Lu, C. Y., Applying TRIZ Systematic Innovation Method to Improve Urinals, Advances in Engineering Research, Vols. 22 (2015), pp. 793-795. 
[7] G. Taguchi, S. Chowdhury, and Y. Wu, "Taguchi's Quality Engineering Handbook”, John Wiley \& Sons, New York, NY, USA, 2004.

[8] Chao-Ton Su, "Quality Engineering", Chinese Society for Quality (2009), Taipei, Taiwan.

[9] Kane, V.E., Process Capability Indices, Journal of Quality Technology, Vol.18, No.1 (1986), pp.41-52. 